\title{
Successful Pregnancy Outcome in a Patient of Chronic Myeloid Leukemia Without Therapy
}

\author{
Roychowdhury Joydeb • Bhattacharyya Maitreyee • \\ Kumar Kundu Arup · Panfalia Madhavi
}

Received: 7 November 2007/Accepted: 20 October 2009/Published online: 26 October 2011

(C) Federation of Obstetric \& Gynecological Societies of India 2011

\section{Introduction}

The real incidence of leukemia during gestation is not well known. It is estimated to range from 1 in 75,000 to 100,000 pregnancies [1]. Acute leukemias are more frequent with pregnancy. Chronic Myeloid Leukemia (CML) is very rare and accounts for less than $10 \%$ of all cases. The management of CML during pregnancy is a difficult problem because of the potential effects of therapy on the fetus. In this circumstance, the physician should not only provide adequate treatment to ensure continued maternal well-being but also avoid fetal compromise. In this case report, we describe a CML patient with pregnancy who delivered a healthy baby at term without any therapy.

Roychowdhury J., Associate Professor .

Panfalia M., Clinical Assistant

Department of Obstetrics \& Gynaecology, Nil Ratan Sircar

Medical College, 138 AJC Bose Road, Kolkata 700 014, India

Bhattacharyya M., Associate Professor

Department of Haematology, Nil Ratan Sircar Medical College,

138 AJC Bose Road, Kolkata 700 014, India

Kumar Kundu A., Associate Professor

Department of Medicine, Nil Ratan Sircar Medical College,

138 AJC Bose Road, Kolkata 700 014, India

Roychowdhury J. ( $₫)$, Associate Professor

BE-177, Sector-1, Salt Lake, Kolkata 700 064, India

e-mail: rcjoydeb@gmail.com

\section{Case Report}

A 30 year old woman with chronic myeloid leukemia attended antenatal clinic of NRS Medical College Kolkata with 32 weeks of pregnancy. The patient was married for, 6 years. She had history of two spontaneous abortions 6 months apart at 12 and 20 weeks of gestation in early years of her married life 4 years before this pregnancy. Following these miscarriages, she was suffering from secondary infertility. Old documents showed that she attended local physician for dragging pain in the left upper abdomen and was found to have moderate splenomegaly. Subsequent peripheral blood and bone marrow examination confirmed it to be a case of Philadelphia chromosome positive CML. She was prescribed hydroxyurea, which she took for 5 months and stopped on her own. She conceived in the next cycle spontaneously. Physical examination showed that she had mild pallor associated with a huge splenomegaly. Uterus was $28-30$ weeks $(4 \mathrm{~cm}$ above the umbilicus) deviated to the right and carrying a small, growth-retarded fetus with a heart rate of $140 / \mathrm{min}$. Opinion of the hematologist was immediately sought for. Complete hemogram showed Hb-9.2 g\%, Hct-28.2\%, MCV-94.3 fl, MCH-33.4 pg/ml, WBC-175 × 10 $3 / \mathrm{I}$, platelets- $601 \times 10^{3} /$ I, and differential count showed P 36 L12 M7 B4 E2, with myelocyte $19 \%$, metamyelocyte $17 \%$, blast cell $3 \%$. Blood biochemistry revealed normal liver function test, and blood sugar, urea, creatinine and uric acid levels were 133, $24,0.8,11 \mathrm{mg} \%$, respectively. She was advised to take hydroxyurea or to undergo leukapheresis till delivery. She 
refused to take any sort of therapy till the pregnancy got over. The patient was kept under close monitoring with weekly blood count and blood biochemistry. Her $\mathrm{Hb} \%$ remained around $9 \mathrm{~g} \%$, WBC count varied between 150 and $200 \times 10^{3} / \mathrm{ml}$, blood biochemistry remained normal except a high uric acid level. She went into labour at 37 weeks and delivered normally a healthy male child of $2.5 \mathrm{~kg}$. On 8th day of puerperium she developed secondary post partum hemorrhage, which required exploration under general anesthesia and blood transfusion. After 1 week she was discharged from the hospital, and asked to attend haematology out patient department and well baby clinic regularly. She was put on hydroxyurea as per advice of the haematologist. Both the mother and the baby are doing well after months of follow-up. Her spleen had regressed completely and she was maintaining a total leucocyte count of 4-5,000 with normal differentials.

\section{Discussion}

The coincidence of CML and pregnancy is an uncommon event, particularly because CML occurs mostly in older age groups. In contrast this patient presented at young age. Pregnancy and cancer is a complex situation. Often treatment cannot be delayed. When chemotherapy is needed urgently, this typically requires termination of pregnancy [2]. Many patients with CML have been reported to have a successful pregnancy. CML has been treated during pregnancy with busulfan, alpha-interferon, hydroxyurea and leukapheresis. Unfortunately, the potential teratogenic effects of chemotherapy on the fetus make their use during pregnancy much less attractive. Indeed, congenital malformations have been associated with busulphan therapy during pregnancy in at least three cases [3]. Teratogenic effects have also been seen in the offspring of rats given five times the normal human dose of hydroxyurea [4] Although, alpha-interferon has not been associated with teratogenicity in either animal studies or in humans on receiving the drug during pregnancy, it has been shown to have abortifacient effects in rhesus monkeys, albeit at doses many times higher than those used to treat patients. Arguably, there are other reports of pregnant patients receiving busulphan, hydroxyurea, or alpha-interferon without apparent teratogenic or abortifacient effects. However, there is paucity of data regarding CML patients on imatinib mesylate becoming pregnant and completing pregnancy. Imatinib has been found to be teratogenic in rats. This patient with two previous fetal losses conceived after a period of secondary infertility refused to take any form of therapy when explained about the potential effect of therapy on the fetus. But fortunately pregnancy without any medication in the antenatal period continued uneventful and had successful outcome. While CML may not need to be treated immediately, and pregnancy does not appear to affect the course of the disease, there is still a risk of leukostasis, as well as the risk of placental insufficiency with consequent below-normal fetal birth weight, increased fetal prematurity, and increased mortality if CML is left untreated through out the pregnancy [9]. Fortunately, the antenatal and intranatal periods of the patient were uneventful and she delivered a healthy baby. The secondary postpartum haemorrhage that she experienced was mainly due to the retained bits of placenta and not related to the disease process.

\section{References}

1. Haas JF. Pregnancy in association with a newly diagnosed cancer: a population-based epidemiologic assessment. Int J Cancer. 1984; 34:229-35.

2. Heartin E, Walkinshaw S, Clark RE. Successful outcome of pregnancy in chronic myeloid leukemia treated with imatinib. Leuk Lymphoma. 2004;45:1307-8.

3. Caligiuri MA, Mayer RJ. Pregnancy and leukemia. Sem Oncol. 1989;16:388-96.

4. Asano Y, Okaniwa A. In utero morphological effects of hydroxyurea on the fetal development in Sprague-Dawley rats. Exp Anim. 1987;36:143-9.

5. Montvale NJ. Physicians desk reference. 51st ed. Montvale: Medical Economics Company, Inc.; 1997. p. 2308-11.

6. Earll JM, May RL. Busulfan therapy of myelocytic leukemia during pregnancy. Am J Obstet Gynecol. 1965;92:580-1.

7. Jackson N, Shukri A, AJi K. Hydroxyurea treatment for chronic myeloid leukaemia during pregnancy. Br J Haematol. 1993;85: 203-4.

8. Crump M, Wang X-H, Sermer M, et al. Successful pregnancy and delivery during alpha-interferon therapy for chronic myeloid leukemia. Am J Hematol. 1992;40:238-9.

9. Juarez S, Cuadrado Pastor JM, Feliu J. Association of leukemia and pregnancy: clinical and obstetric aspects. Am J Clin Oncol. 1988;II:159-65. 